\title{
Avaliação in vitro dos efeitos do óleo da semente de Carapa guianensis Aubl. sobre larvas de nematóides gastrintestinais de caprinos e ovinos
}

\author{
FARIAS, M.P.O. ${ }^{1 *}$; TEIXEIRA, W.C. ${ }^{1}$; WANDERLEY, A.G. ${ }^{2}$; ALVES, L.C. ${ }^{1}$; FAUSTINO, M.A.G. ${ }^{1}$ \\ ${ }^{1}$ Universidade Federal Rural de Pernambuco, Departamento de Medicina Veterinária, Rua Dom Manoel de Medeiros \\ s/n, Dois Irmãos, CEP: 52171-900, Recife-Brasil *marciapbo@gmail.com 2'Universidade Federal de Pernambuco, \\ Departamento de Fisiologia e Farmacologia, Av. Prof. Moraes Rego, 1235, Cidade Universitária, CEP: 50670-901, \\ Recife-Brasil
}

RESUMO: Com objetivo de avaliar in vitro a ação do óleo da semente da Carapa guianensis (Andiroba) no cultivo de larvas de nematóides gastrintestinais de animais das espécies caprina e ovina, foram testadas cinco diluições do óleo de andiroba (100, 50, 30, 25 e 10\%), com três repetições por tratamento, utilizando-se tween 80 como dispersante, formando-se ainda três grupos controle, um controle negativo (água destilada), outro controle negativo (água destilada + tween 80) e um controle positivo (Doramectina). A atividade da andiroba sobre os ovos de nematóides gastrintestinais foi determinada pelo cálculo dos percentuais de redução de larvas por gramas de fezes. Os resultados revelaram na espécie caprina redução altamente efetiva no número de larvas totais para os tratamentos 100, 50 e 30\% com médias nulas para todos os gêneros de nematóides. Na espécie ovina observou-se redução altamente efetiva no número de larvas totais em todos os tratamentos, com médias nulas nos tratamentos 100,50 e $30 \%$. Os resultados obtidos neste experimento demonstram que o óleo da semente de Carapa guianensis possui atividade in vitro contra larvas de nematóides gastrintestinais de caprinos e ovinos.

Palavras-chave: anti-helmíntico, plantas medicinais, ruminantes

\begin{abstract}
In vitro evaluation of Carapa guianensis Aubl. seed oil effects on larvae from gastrointestinal nematodes of goats and sheep. This study aimed to evaluate the in vitro action of Carapa guianensis (Andiroba) seed oil on the cultivation of larvae from gastrointestinal nematodes of goats and sheep. Five andiroba oil dilutions (100, 50, 30, 25, and 10\%) were assayed, with three replicates per treatment, using Tween 80 as surfactant. Three control groups were formed: a negative control (distilled water), another negative control (distilled water + Tween 80 ) and a positive control (Doramectin). The activity of andiroba on the eggs from gastrointestinal nematodes was obtained by calculating larva reduction percentages per gram of feces. In goats, a highly effective reduction in the total number of larvae was detected for treatments 100, 50 and $30 \%$, with null means for all nematode genera. In sheep, a highly effective reduction in the total number of larvae was observed for all treatments, with null means for treatments 100,50 and $30 \%$. Such results indicate that Carapa guianensis (Andiroba) seed oil has in vitro activity against larvae from gastrointestinal nematodes of goats and sheep.
\end{abstract}

Key words: anthelmintic, medicinal plants, ruminants

\section{INTRODUÇÃO}

O parasitismo por nematóides gastrintestinais é responsável por elevadas perdas econômicas na caprinocultura e ovinocultura, tendo distribuição geográfica ampla, comprometendo a rentabilidade desse sistema pecuário no Nordeste brasileiro (Vieira et al., 1992; Pinheiro et al., 2000; Melo et al., 2003). Segundo Amarante et al. (2004), $100 \%$ dos ruminantes domésticos são portadores de pelo menos uma espécie de endoparasita.

O controle da infecção por nematóides gastrintestinais ainda é essencialmente químico (Costa \& Vieira, 1984; Charles, 1989), porém o rápido aparecimento da resistência associado ao alto custo do tratamento e a determinação de problemas relacionados com os resíduos químicos nos produtos de origem animal e contaminação ambiental

Recebido para publicação em 06/05/09

Aceito para publicação em 06/01/10

Rev. Bras. PI. Med., Botucatu, v.12, n.2, p.220-226, 2010. 
incentivaram a pesquisa de alternativas de controle, como o estudo dos fitoterápicos (Waller et al., 1995; Chagas et al., 2002; Vieira, 2006).

Várias plantas com atividade anti-helmíntica foram relatadas em diversas partes do mundo (Menezes et al.,1992; Assis, 2000; Pessoa, 2001; Almeida et al., 2003; Batista et al., 2003; Batatinha et al., 2004; Borba \& Amorim, 2004; Batatinha et al., 2005; Furtado et al., 2005; Girão et al., 2005; Costa et al., 2006). Trabalhos para comprovação da ação sobre os parasitos podem oferecer novas alternativas de controle efetivo e econômico (Chagas, 2004).

Os extratos de plantas são utilizados pelo homem desde nossos ancestrais e ainda hoje os produtores rurais utilizam várias plantas empiricamente no tratamento das parasitoses dos animais domésticos (Batatinha et al., 2004; Chagas, 2004), sendo, necessária realização de testes laboratoriais para se determinar a possibilidade de uso de determinado fitoterápico (Chagas, 2004). Segundo Borges (2003), o método in vitro é rápido e aplicável na determinação da ação ovicida e larvicida de anti-helmíntico. Permite avaliação preliminar da existência de propriedades antihelmínticas logo, é o primeiro passo para caracterização dos possíveis compostos ativos presentes nos extratos vegetais (Costa et al., 2006).

A Carapa guianensis (andiroba), espécie botânica da família Meliaceae, ocorre naturalmente, formando associações na América Central, Peru, Brasil, Suriname, Guianas, África Tropical, Antilhas, Colômbia e Venezuela. Encontra-se também com muita freqüência nas vertentes das colinas, em solos bem drenados. É muito cultivada em terra firme, onde atinge menor porte (Loureiro et al., 1979). No Brasil, ocorre em toda bacia amazônica, prefere as várzeas e faixas alagáveis ao longo dos cursos d'água (Azevedo et al., 1997). Encontrada do Pará à Bahia, apresenta características silviculturais de bom desenvolvimento. É madeira de alto valor econômico utilizada principalmente em construção naval, carpintaria, marcenaria, confecção de portas e caixotaria, e a semente encerra $70 \%$ de óleo insetífugo e medicinal que é utilizado na medicina popular da região norte do Brasil como febrífugo, anti-reumático, antibacteriano e repelente de insetos (Loureiro et al., 1979; Lorenzi, 1992; Neves et al., 2004), no entanto nada ainda se sabe sobre a bioatividade em helmintos.

Desenvolveu-se este trabalho com o objetivo de avaliar in vitro o efeito ovicida do óleo da semente da C. guianensis em culturas de larvas de nematóides gastrintestinais de caprinos e ovinos, a fim de se obterem subsídios para novos estudos sobre o uso no controle dessas parasitoses.

\section{MATERIAL E MÉTODO}

Utilizou-se extrato hexânico da semente de Carapa guianensis, cuja extração foi realizada por percolação a frio, com oito litros de solvente polar (98\% Etanol) para cada amostra extraída, processada na Universidade Federal do Pará, na Central de Extração do Departamento de Química. As sementes foram coletadas no município de Curuçá-Pará atendendo os protocolos para obtenção de patentes. As exsicatas foram depositadas no herbário do Museu Paraense Emilio Goeldi (Instituição credenciada pelo IBAMA). As coletas foram feitas de acordo com a legislação de acesso ao Patrimônio Genético Nacional (MP № 2.186-16 de 23/08/2001). Foi utilizada a licença de acesso do Convênio UFPA/ Extracta Moléculas Naturais S.A.

Foram utilizadas amostras fecais de caprinos do município de Gravatá, mesorregião do Agreste do estado de Pernambuco e de ovinos pertencentes ao Departamento de Medicina Veterinária (DMV) - Universidade Federal Rural de Pernambuco, as quais foram coletadas diretamente da ampola retal de animais naturalmente infectados por helmintos, utilizando-se sacos plásticos. As amostras foram acondicionadas em caixas isotérmicas, contendo gelo reciclável e encaminhadas ao Laboratório de Doenças Parasitárias dos Animais Domésticos - Área de Medicina Veterinária Preventiva do Departamento de Medicina Veterinária Universidade Federal Rural de Pernambuco, onde foram processadas para determinação do número de ovos por grama de fezes (OPG), segundo técnica de Gordon \& Whitlock (1939), interpretando-se o grau de infecção segundo Ueno \& Gonçalves (1998). De cada espécie animal, foram selecionadas as amostras fecais, formando-se um homogeneizado para realização dos cultivos de larvas, segundo técnica descrita por Robert \& O'Sullivan (1950).

Para a obtenção do total de larvas por grama de fezes (LPG) foram pesados $3 \mathrm{~g}$ de fezes, misturando-se a $2 \mathrm{~g}$ de vermiculita, separadamente para cada espécie hospedeira. Cada cultivo, assim preparado, foi submetido a cinco concentrações do óleo da semente de Carapa guianensis (100, 50, 30, 25 e $10 \%$ ), correspondendo aos tratamentos T1, T2, T3, T4 e T5, utilizando tween 80 como dispersante, com três repetições por tratamento, formando-se, ainda, três grupos controles, sendo dois controles negativos, um com água destilada (C1) e outro com água destilada + tween 80 (C2) e, ainda, um controle positivo (Cp) para o qual se empregou a Doramectina.

Os cultivos foram mantidos em temperatura ambiente no laboratório durante oito dias, decorridos os quais, as larvas infectantes de nematóides gastrintestinais foram coletadas em tubos de ensaio 
e armazenadas sob refrigeração até o momento de identificação por gênero, segundo Ueno \& Gonçalves (1998). Para contagem e identificação das larvas, retirou-se alíquota de $0,25 \mathrm{~mL}$ deste material com auxílio de pipeta, colocando em lâmina de microscopia, adicionando-se, em seguida uma gota de lugol e cobrindo com lamínula, totalizando cinco lâminas para cada tratamento, sendo então examinadas em microscópio óptico.

A atividade da Carapa guianensis sobre os ovos de nematóides gastrintestinais foi determinada pelo cálculo dos percentuais de redução de larvas por gramas de fezes (LPG), utilizando-se a fórmula abaixo descrita (Vizard \& Wallace, 1987).

$$
R=100 .(1-T / C)
$$

$\mathrm{R}=$ Redução calculada no LPG

$T=$ Média geométrica dos LPGs $(L P G+10)$ dos cultivos tratados

$C=$ Média geométrica dos LPGs $($ LPG +10$)$ dos cultivos não tratados

A atividade in vitro do óleo da semente de Carapa guianensis foi considerada segundo a classificação do índice de eficácia proposto pela Associação Mundial para Avanço da Parasitologia Veterinária (W.A.A.V.P.) (Powers et al., 1982), em que se determina que um produto é altamente efetivo se apresentar mais de $90 \%$ de ação contra o parasito tratado; moderadamente efetivo quando atuar entre 80 e $90 \%$, pouco efetivo quando a ação esteja entre 60 e $70 \%$ e não efetivo abaixo de $60 \%$.

Para análise dos dados foi obtida a média aritmética e o desvio padrão do número de larvas e utilizado o teste $\mathrm{F}$ (ANOVA) para a referida variável em cada gênero de larva e no caso de diferença significativa foram utilizados os testes de comparações pareadas de Tukey (Zar, 1999). Considerou-se, nas decisões estatísticas, o nível de significância de 5,0\%, utilizando-se o "software" SAS (Statistical Analysis System) na versão 8.0 para microcomputador.

\section{RESULTADO E DISCUSSÃO}

Os percentuais de redução do número de larvas nos cultivos de fezes de animais da espécie caprina e ovina após tratamento com óleo da semente de Carapa guianensis encontram-se nas Tabelas 1 a 4.

Os cultivos de larvas de nematóides gastrintestinais da espécie caprina nos grupos controles (C1 e C2) revelaram larvas infectantes do gênero Haemonchus, Oesophagostomum e Trichostrongylus, com predominância do gênero Haemonchus, estando de acordo com os mais frequentes obtidos por Almeida et al. (2003), e níveis baixos de infecção do gênero Trichostrongylus, conforme os dados obtidos por Almeida et al. (2003; 2004) e Batatinha et al. (2004).

Analisando o percentual de redução na espécie caprina, considerando o controle negativo C1, foi demonstrada redução altamente efetiva para os tratamentos T1, T2, T3 e Controle positivo (Cp) para os gêneros Haemonchus e Oesophagostomum, e no número total de larvas e moderadamente efetiva para o gênero Trichostrongylus em todos os tratamentos (Tabela 1). Almeida et al. (2003) e Batatinha et al. (2004) relataram eficácia elevada para o total de larvas de Haemonchus e Oesophagostomum em cultivos de larvas tratados com Cymbopogon citratus (capimsanto), Digitaria insulares (capim-açu), Carica papaya (mamão) e Musa cavendishii (banana), no entanto, nenhum relato existe em literatura sobre Carapa guianensis.

Constatou-se, no presente estudo, na espécie ovina nos cultivos de larvas de nematóides gastrintestinais nos grupos controles (C1 e C2) larvas infectantes do gênero Haemonchus, Oesophagostomum, Trichostrongylus e Strongyloides, com predominância do gênero

TABELA 1. Percentual de redução do número de larvas de terceiro estágio de nematóides gastrintestinais de caprinos após o tratamento de coproculturas com óleo da semente de andiroba em relação ao controle negativo $\mathrm{C} 1$.

\begin{tabular}{ccccc}
\hline & \multicolumn{3}{c}{ Gêneros } & \\
\cline { 2 - 4 } Tratamento & Haemonchus & Oesophagostomum & Trichostrongylus & Total \\
\hline T1 & 94,24 & 91,72 & 86,50 & 91,55 \\
T2 & 94,24 & 91,72 & 86,50 & 91,55 \\
T3 & 94,24 & 91,72 & 86,50 & 91,55 \\
T4 & 92,12 & 90,32 & 84,22 & 89,33 \\
T5 & 89,59 & 87,22 & 81,54 & 86,38 \\
Cp & 94,24 & 91,72 & 86,50 & 91,55 \\
\hline
\end{tabular}

C1- controle negativo água destilada, T1- tratamento 100\% óleo andiroba, T2- tratamento 50\% óleo andiroba, T3- tratamento $30 \%$ óleo andiroba, T4- tratamento $25 \%$ óleo andiroba, T5- tratamento $10 \%$ óleo andiroba e Cp- controle positivo (Doramectina)

Rev. Bras. Pl. Med., Botucatu, v.12, n.2, p.220-226, 2010. 
Haemonchus.

Considerando o controle negativo $\mathrm{C} 1$ na espécie ovina, verificou-se percentual de redução altamente efetivo no número total de larvas em todos os tratamentos. Entretanto, com relação aos gêneros de nematóides, esta atividade em todos os tratamentos, demonstrou pouco efetiva para Strongyloides (Tabela 2).
Com relação ao controle negativo $\mathrm{C} 2$ na espécie caprina e ovina, foi observado que o dispersante utilizado (Tween 80 ) não acarretou nenhuma interferência no percentual de redução de larvas de nematóides gastrintestinais nas espécies animais, constatando em geral o mesmo padrão de resultados para os percentuais de redução com os controles $\mathrm{C} 1$ e $\mathrm{C} 2$, tanto para

TABELA 2. Percentual de redução do número de larvas de terceiro estágio de nematóides gastrintestinais de ovinos após o tratamento de coproculturas com óleo da semente de Andiroba em relação ao controle negativo $\mathrm{C} 1$.

\begin{tabular}{|c|c|c|c|c|c|}
\hline \multirow[b]{2}{*}{ Tratamento } & \multicolumn{4}{|c|}{ Gêneros } & \multirow[b]{2}{*}{ Total } \\
\hline & Haemonchus & Oesophagostomum & Trichostrongylus & Strongyloides & \\
\hline T1 & 94,34 & 92,18 & 84,79 & 79,77 & 98,39 \\
\hline T2 & 94,34 & 92,18 & 84,79 & 79,77 & 98,39 \\
\hline T3 & 91,28 & 90,86 & 84,79 & 76,36 & 93,36 \\
\hline $\mathrm{T} 4$ & 90,47 & 87,93 & 82,22 & 72,35 & 91,39 \\
\hline T5 & 94,34 & 92,18 & 84,79 & 79,77 & 98,39 \\
\hline $\mathrm{Cp}$ & 94,34 & 92,18 & 84,79 & 79,77 & 98,39 \\
\hline
\end{tabular}

C1- controle negativo água destilada, T1- tratamento 100\% óleo andiroba, T2- tratamento 50\% óleo andiroba, T3- tratamento $30 \%$ óleo andiroba, T4- tratamento $25 \%$ óleo andiroba, T5- tratamento $10 \%$ óleo andiroba, Cp controle positivo (Doramectina)

TABELA3. Percentual de redução do número de larvas de terceiro estágio de nematóides gastrintestinais de caprinos após o tratamento de coproculturas com óleo da semente de Andiroba em relação ao controle negativo C2.

\begin{tabular}{ccccc}
\hline & \multicolumn{3}{c}{ Gêneros } & \\
\cline { 2 - 4 } Tratamento & Haemonchus & Oesophagostomum & Trichostrongylus & Total \\
\hline T1 & 92,71 & 91,52 & 81,89 & 89,89 \\
T2 & 92,71 & 91,52 & 81,89 & 89,89 \\
T3 & 92,71 & 91,52 & 81,89 & 89,89 \\
T4 & 90,04 & 90,08 & 78,89 & 87,23 \\
T5 & 86,84 & 86,90 & 72,25 & 83,70 \\
Cp & 92,71 & 91,52 & 81,89 & 89,89 \\
\hline
\end{tabular}

C2- controle negativo água + tween 80 , T1- tratamento 100\% óleo andiroba, T2- tratamento 50\% óleo andiroba, T3- tratamento $30 \%$ óleo andiroba, T4- tratamento $25 \%$ óleo andiroba, T5- tratamento $10 \%$ óleo andiroba e Cp- controle positivo (Doramectina)

TABELA 4. Percentual de redução do número de larvas de terceiro estágio de nematóides gastrintestinais de ovinos após o tratamento de coproculturas com óleo da semente de Andiroba considerando controle negativo C2.

\begin{tabular}{cccccc}
\hline & \multicolumn{5}{c}{ Gênero } \\
\cline { 2 - 5 } Tratamento & Haemonchus & Oesophagostomum & Trichostrongylus & Strongyloides & Total \\
\hline T1 & 94,12 & 91,85 & 81,28 & 61,22 & 92,60 \\
T2 & 94,12 & 91,85 & 81,28 & 61,22 & 92,60 \\
T3 & 94,12 & 91,85 & 81,28 & 61,22 & 92,60 \\
T4 & 90,93 & 90,48 & 81,28 & 54,67 & 82,93 \\
T5 & 90,09 & 87,43 & 78,12 & 46,99 & 18,87 \\
Cp & 94,12 & 91,85 & 81,28 & 61,22 & 92,60 \\
\hline
\end{tabular}

C2- controle negativo água destilada + tween 80 , T1- tratamento $100 \%$ óleo andiroba, T2- tratamento $50 \%$ óleo andiroba, T3- tratamento $30 \%$ óleo andiroba, T4- tratamento $25 \%$ óleo andiroba, T5- tratamento $10 \%$ óleo andiroba e Cp- controle positivo (Doramectina) 
TABELA5. Média aritmética e desvio padrão do número de larvas de terceiro estágio de nematóides gastrintestinais obtidos de coproculturas de caprinos tratadas com óleo da semente de Andiroba.

\begin{tabular}{|c|c|c|c|c|}
\hline \multirow[b]{2}{*}{ Tratamento ${ }^{(1)}$} & \multicolumn{3}{|c|}{ Gênero } & \multirow[b]{2}{*}{ Total } \\
\hline & Haemonchus & Oesophagostomum & Trichostrongylus & \\
\hline C1 & $171,67 \pm 65,01^{(\mathrm{A})}$ & $113,67 \pm 24,38^{(\mathrm{A})}$ & $64,67 \pm 11,72^{(\mathrm{A})}$ & $350,00 \pm 48,66^{(A)}$ \\
\hline $\mathrm{C} 2$ & $130,67 \pm 37,11^{\text {(A) }}$ & $112,33 \pm 38,44^{(\mathrm{A})}$ & $46,33 \pm 13,50^{(\mathrm{A})}$ & $289,33 \pm 49,24^{(A)}$ \\
\hline $\mathrm{Cp}$ & $0,00 \pm 0,00^{(B)}$ & $0,00 \pm 0,00^{(B)}$ & $0,00 \pm 0,00^{(B)}$ & $0,00 \pm 0,00^{(B)}$ \\
\hline $\mathrm{T} 1$ & $0,00 \pm 0,00^{(B)}$ & $0,00 \pm 0,00^{(B)}$ & $0,00 \pm 0,00^{(B)}$ & $0,00 \pm 0,00^{(B)}$ \\
\hline T2 & $0,00 \pm 0,00^{(B)}$ & $0,00 \pm 0,00^{(B)}$ & $0,00 \pm 0,00^{(B)}$ & $0,00 \pm 0,00^{(B)}$ \\
\hline T3 & $0,00 \pm 0,00^{(B)}$ & $0,00 \pm 0,00^{(B)}$ & $0,00 \pm 0,00^{(B)}$ & $0,00 \pm 0,00^{(B)}$ \\
\hline $\mathrm{T} 4$ & $4,00 \pm 3,46^{(B)}$ & $2,00 \pm 3,46^{(B)}$ & $2,00 \pm 3,46^{(B)}$ & $8,00 \pm 9,17^{(B)}$ \\
\hline T5 & $6,33 \pm 6,51^{(B)}$ & $6,33 \pm 6,51^{(\mathrm{B})}$ & $4,00 \pm 3,46^{(B)}$ & $16,67 \pm 9,71^{(\mathrm{B})}$ \\
\hline Valor $p$ & $\mathrm{p}^{(2)}<0,0001^{*}$ & $\mathrm{p}^{(2)}<0,0001^{*}$ & $\mathrm{p}^{(2)}<0,0001^{*}$ & $\mathrm{p}^{(2)}<0,0001^{*}$ \\
\hline
\end{tabular}

$\left({ }^{*}\right)$ - Diferença significante a 5,0\%. (1) C1 controle água destilada, C2 controle água destilada + Tween 80 , Cp controle positivo (Doramectina), T1- tratamento $100 \%$ óleo andiroba, T2- tratamento 50\% óleo andiroba, T3- tratamento $30 \%$ óleo andiroba, T4- tratamento $25 \%$ óleo andiroba, T5- tratamento $10 \%$ óleo andiroba (2) - Através do teste F (ANOVA). Obs: Se nenhuma letra entre parêntesis for comum existe diferença significante entre os tratamentos correspondentes através das comparações pareadas de Tukey.

caprinos (Tabelas 1 e 3 ) quanto para ovinos (Tabelas 2 e 4).

Na espécie caprina, analisando-se as médias aritméticas do número de larvas, obtiveram-se médias nulas para os tratamentos T1, T2 e T3 para todos os gêneros. Nos tratamentos T4 e T5, embora as médias não tenham sido nulas, os números médios de larvas foram significativamente semelhantes aos tratamentos T1, T2 e T3. Os testes estatísticos também demonstraram diferenças significativas entre os tratamentos ao nível de significância de $(p<0,05)$ em cada um dos gêneros e no total (Tabela 5).

TABELA 6. Média aritmética e desvio padrão do número de larvas de terceiro estágio de nematóides gastrintestinais obtido de coproculturas de ovinos tratadas com óleo da semente de Andiroba.

\begin{tabular}{|c|c|c|c|c|c|}
\hline \multirow[b]{2}{*}{ Tratamento $^{(1)}$} & \multicolumn{4}{|c|}{ Gêneros } & \multirow[b]{2}{*}{ Total } \\
\hline & Haemonchus & Oesophagostomum & Trichostrongylus & Strongyloides & \\
\hline $\mathrm{C} 1$ & $167,33 \pm 14,74^{(A)}$ & $120,33 \pm 29,14^{(A)}$ & $56,00 \pm 7,00^{(A)}$ & $42,67 \pm 21,20^{(A)}$ & $386,33 \pm 30,86^{(A)}$ \\
\hline $\mathrm{C} 2$ & $160,33 \pm 9,29^{(A)}$ & $116,67 \pm 37,50^{(\mathrm{A})}$ & $43,67 \pm 6,03^{(B)}$ & $16,33 \pm 6,51^{(\mathrm{B})}$ & $337,00 \pm 52,56^{(A)}$ \\
\hline $\mathrm{Cp}$ & $00,00 \pm 0,00^{(B)}$ & $00,00 \pm 0,00^{(B)}$ & $00,00 \pm 0,00^{(\mathrm{C})}$ & $00,00 \pm 0,00^{(B)}$ & $0,00 \pm 0,00^{(\mathrm{B})}$ \\
\hline $\mathrm{T} 1$ & $00,00 \pm 0,00^{(B)}$ & $00,00 \pm 0,00^{(B)}$ & $00,00 \pm 0,00^{(\mathrm{C})}$ & $00,00 \pm 0,00^{(B)}$ & $0,00 \pm 0,00^{(B)}$ \\
\hline $\mathrm{T} 2$ & $00,00 \pm 0,00^{(B)}$ & $00,00 \pm 0,00^{(B)}$ & $00,00 \pm 0,00^{(\mathrm{C})}$ & $00,00 \pm 0,00^{(B)}$ & $0,00 \pm 0,00^{(\mathrm{B})}$ \\
\hline T3 & $00,00 \pm 0,00^{(B)}$ & $00,00 \pm 0,00^{(B)}$ & $00,00 \pm 0,00^{(C)}$ & $00,00 \pm 0,00^{(B)}$ & $0,00 \pm 0,00^{(B)}$ \\
\hline $\mathrm{T} 4$ & $6,33 \pm 6,51^{(\mathrm{B})}$ & $2,00 \pm 3,46^{(B)}$ & $00,00 \pm 0,00^{(\mathrm{C})}$ & $2,00 \pm 3,46^{(B)}$ & $10,33 \pm 13,05^{(B)}$ \\
\hline T5 & $8,67 \pm 10,266^{(B)}$ & $6,33 \pm 6,51^{(\mathrm{B})}$ & $2,00 \pm 3,46^{(C)}$ & $4,00 \pm 3,46^{(B)}$ & $21,00 \pm 22,65^{(\mathrm{B})}$ \\
\hline Valor $p$ & $\mathrm{p}^{(2)}<0,0001^{*}$ & $\mathrm{p}^{(2)}<0,0001^{*}$ & $\mathrm{P}^{(2)}<0,0001^{*}$ & $\mathrm{p}^{(2)}<0,0001^{*}$ & $\mathrm{P}^{(2)}<0,0001^{*}$ \\
\hline
\end{tabular}

$\left({ }^{*}\right)$ - Diferença significante a 5,0\%. (1) C1 controle água destilada, C2 controle água destilada + Tween 80, Cp controle positivo (Doramectina), T1- tratamento $100 \%$ óleo andiroba, T2- tratamento $50 \%$ óleo andiroba, T3- tratamento $30 \%$ óleo andiroba, T4- tratamento $25 \%$ óleo andiroba, T5- tratamento $10 \%$ óleo andiroba (2) - Através do teste F (ANOVA). Obs: Se nenhuma letra entre parêntesis for comum existe diferença significante entre os tratamentos correspondentes através das comparações pareadas de Tukey. 
Na análise das médias aritméticas do número de larvas de nematóides gastrintestinais em coproculturas de ovinos submetidas aos tratamentos com óleo da semente de Carapa guianensis, foram demonstradas médias nulas para os tratamentos $\mathrm{T} 1$, T2 e T3 para todos os gêneros. Observou-se diferença significativa entre o controle $\mathrm{C} 1$ e todos os tratamentos ao nível de significância de 5,0\% $(p<0,05)$ em cada um dos gêneros de nematóides e no total (Tabela 6).

Apesar de não existirem diferenças significativas entre as médias do número de larvas obtidos nas diferentes concentrações do óleo da semente de Carapa guianensis tanto para caprinos (Tabela 5) como para ovinos (Tabela 6), os percentuais de redução calculados demonstraram haver uma atuação diferenciada das concentrações menores (25 e 10\%), em relação às maiores em praticamente todas as situações analisadas neste estudo (Tabelas 1 a 4).

O óleo da semente de Carapa guianensis, principalmente nas concentrações de 100\% (T1), 50\% (T2) e 30\% (T3), mostrou-se similar à Doramectina (Cp) nos percentuais de redução calculados, com valores altamente efetivos em todos os casos, assim como na inibição completa da eclosão in vitro dos ovos de nematóides. Por ser a Doramectina uma droga pertencente ao grupo da lactonas macrocíclicas, muito utilizada no controle das helmintoses gastrintestinais (Mello et al., 2006) e o fenômeno da resistência anti-helmíntica a este grupo de drogas ser citado em vários países (Waller, 1997), o resultado obtido com o óleo da semente de Carapa guianensis deve ser considerado na possibilidade de indicação deste fitoterápico como fonte alternativa para o tratamento e prevenção da infecção por nematóides gastrintestinais nas espécies estudadas.

A atividade ovicida contra nematóides gastrintestinais de caprinos e ovinos in vitro, demonstrada pelo óleo da semente de Carapa guianensis evidencia a atividade anti-helmíntica deste fitoterápico e a possibilidade como alternativa para o controle de nematóides gastrintestinais de caprinos e ovinos.

\section{REFERÊNCIA}

ALMEIDA, M.A. et al. Avaliação in vitro dos efeitos do extrato alcoólico e do suco de alho (Allium sativum L.) sobre nematódeos gastrintestinais de caprinos. Ciência Veterinária nos Trópicos, v.7, n.1, p.36-43, 2004. ALMEIDA, M.A.O. et al. Efeitos dos extratos aquosos de folhas de Cymbopogon citratus (DC.) Stapf (capim-santo) e de Digitaria inularis (L.) Fedde (capim-açu) sobre cultivos de larvas de nematóides gastrintestinais de caprinos. Revista Brasileira de Parasitologia Veterinária, v.12, n.3, p.125-9, 2003.

AMARANTE, A.F.T. et al. Resistance of Santa Inês, Suffolk and ile de France sheep to naturally acquired gastrointestinal nematode infections. Veterinary Parasitology, v.120, p.91-106, 2004.

ASSIS, L.M. Atividade anti-helmíntica in vitro de extratos de Spigelia anthelmia sobre Haemonchus contortus. 2000. 44p. Dissertação (Mestrado em Ciências Veterinárias) - Departamento de Medicina Veterinária, Universidade Estadual do Ceará, Fortaleza. AZEVEDO, C.P. et al. Formação de mudas de andiroba (Carapa guianensis Aubl. - Meliaceae): 1- Resposta a diferentes níveis de sombreamento. Revista Ciências Agrárias, v.6, n.2, p.1-12, 1997.

BATATINHA, M.J.M. et al. Efeitos do suco de alho (Allium sativum Linn.) em caprinos infectados com nematódeos gastrintestinais: aspectos clínicos. Revista Brasileira de Ciência Veterinária, v.27, n.2, p.18-21, 2005.

BATATINHA, M.J.M. et al. Efeitos dos extratos de folhas de Musa cavendishii Linn. e de sementes de Carica papaya Linn. sobre culturas de larvas de nematódeos gastrintestinais de caprinos. Revista Brasileira de Plantas Medicinais, v.7, n.1, p.11-5, 2004.

BATISTA, L.M. et al. Atividade ovicida e larvicida in vitro de Spigelia anthelmia e Momordica charantia contra o nematódeo Haemonchus contortus. Ciência Animal, v.9, p.67-73, 2003.

BORBA, H.R.; AMORIM, A. Avaliação da atividade de extrato aquoso de Chenopodium ambrosioides L. (ervade-santa-maria) em camundongos naturalmente infectados com Syphacia obvelata e Aspiculurus tetraptera. Revista Brasileira de Parasitologia Veterinária, v.4, n.13, p.133-6, 2004.

BORGES, C.C.L. Atividade in vitro de anti-helmínticos sobre larvas infectantes de nematódeos gastrintestinais de caprinos, utilizando a técnica de coprocultura quantitativa (Ueno, 1995). Parasitologia Latinoamericana, v.58, n.1, p.42-7, 2003.

CHAGAS, A.C.S. Controle de parasitas utilizando extratos vegetais. Revista Brasileira de Parasitologia Veterinária, v.13, p.156-60, 2004.

CHAGAS, A.C.S. et al. Ação larvicida de derivados arilsulfonílicos da (+) cânfora e da (+) isopinocanfona sobre o carrapato Boophilus microplus. Arquivo Brasileiro de Medicina Veterinária e Zootecnia, v.54, n.5, p.462-77, 2002.

CHARLES, T.P. Seasonal prevalence of gastrointestinal nematodes of goats in Pernambuco state, Brazil. Veterinary Parasitology, v.30, p.335-43, 1989.

COSTA, C.T.C. et al. Anthelmintic activity of Azadirachta indica $A$. Juss against sheep gastrointestinal nematodes. Veterinary Parasitology, v.137, p.306-10, 2006.

COSTA, H.M.A.; VIEIRA, L.S. Endoparasitos permanentes de caprinos e ovinos em Sobral - CE. Pesquisa Agropecuária Brasileira, v.19, p.639-46, 1984.

FURTADO, S.K. et al. Efeito de Carica papaya L. (Caricaceae) e Musa paradisíaca Linn. (Musaceae) sobre o desenvolvimento de ovos de nematódeos gastrintestinais de ovinos. Arquivo do Instituto Biológico, v.72, n.2, p.191-7, 2005.

GIRÃO, E.S. et al. Identificação e avaliação de plantas medicinais com efeito anti-helmínticos em caprinos. Disponível em: <http://www. sbz. org.br. br/ eventos / fortaleza / SOSTPROD \% 5csbz 438. pdf. > Acesso em:

Rev. Bras. PI. Med., Botucatu, v.12, n.2, p.220-226, 2010. 
11 set. 2005.

GORDON, H.McL.; WHITLOCK, H.V. A new technique for counting nematoda eggs in sheep faeces. Journal Commonwealth Science and Industry Organization, v.12, n.1, p.50-2, 1939.

LORENZI, H. Árvores brasileiras: manual de identificação e cultivo de plantas arbóreas nativas do Brasil. Nova Odessa: Instituto Plantarum,1992. 368p. LOUREIRO, A.A.; SILVA, M.F.; ALENCAR, J.C. Essências madeireiras da Amazônia. Manaus: INPA/ SUFRAMA, U.A., 1979. 245p.

MELLO, M.H.A. et al. Resistência lateral às macrolactonas em nematodas de bovinos. Archives of Veterinary Science, v.11, n.1, p.8-12, 2006.

MELO, L.M. et. al. Passagem do fungo Monacrosporium thaumasium através do trato gastrointestinal de caprinos e atividade predatória contra Haemonchus contortus. Ciência Rural, v.33, n.1, p.169-71, 2003.

MENEZES, R.C.A. A. et al. Estudos preliminares in vitro da atividade ovicida de folhas e sementes de quatro leguminosas sobre Haemonchus contortus de caprinos. Arquivo da Universidade Federal do Rio de Janeiro, v.15, n.2, p.121-7, 1992.

NEVES, O.S.C. et al. Crescimento, produção de matéria seca e acúmulo de $\mathrm{N}, \mathrm{P}, \mathrm{K}, \mathrm{Ca}, \mathrm{Mg}$, e $\mathrm{S}$ na parte aérea de mudas de andiroba (Carapa guianensis Aubl.) cultivadas em solo de várzea, em função de diferentes doses de fósforo. Revista Árvore, v.28, n.3, p.343-9, 2004.

PESSOA, L.M. Atividade ovicida in vitro da plantas medicinais contra Haemonchus contortus. 2001. 68p. Dissertação (Mestrado em Ciência Veterinária)Departamento de Medicina Veterinária, Universidade Estadual do Ceará, Fortaleza.

PINHEIRO, R.R. et al. Aspectos epidemiológicos da caprinocultura cearence. Arquivo Brasileiro de Medicina
Veterinária e Zootecnia, v.52, n.5, p.20-2, 2000.

POWERS, K.G. et al. World Association for the Advancement of Veterinary Parasitology (W.A.A.V.P.) Guidelines for evaluating the efficacy of anthelmintics in ruminants (bovine and ovine). Veterinary Parasitology, v.10, p.265-84, 1982.

ROBERTS, F.H.S.; O'SULLIVAN, J.P. Methods for egg counts and larval cultures for strongyles infesting the gastrointestinal tract of cattle. Journal of Agricultural Research, v.1, p.99-102, 1950.

UENO, H.; GONÇALVES, P.C. Manual para diagnóstico das helmintoses de ruminantes. 4.ed. Tokyo: Japan International Cooperation Agency, 1998. 143p.

VIEIRA, L.S. Endoparasitoses gastrintestinais de caprinos e ovinos: Alternativas de controle. In: ENCONTRO NACIONAL DE PRODUÇÃO DE CAPRINOS E OVINOS, 1., 2006, Campina Grande. Anais... João Pessoa, 2006. 1 CD-ROM.

VIEIRA, L.S.; BERNE, M.E.A.; CAVALCANTE, A.C.R. Haemonchus contortus resistance to ivermectin and netobini in Brazilian sheep. Veterinary Parasitology, v.45, p.111-6, 1992.

VIZARD, A.L.; WALLACE, R.J. A simplified egg count reduction test. Australian Veterinary Journal, v.64, n.4, p.109-11, 1987.

WALLER, P.J. Nematode parasite control of livestock in the tropics / subtropics: the need for novel approaches. International Journal for Parasitology, v.27, n.10, p.1193-201, 1997.

WALLER, P.J. et al. Anthelmintic resistance in nematode parasites of sheep: learning from the Australian experience. Veterinary Record, v.136, p.411-3, 1995.

ZAR, J.H. Biostatistical analysis. 4.ed. New Jersey: Prentice Hall, 1999. 929p. 